\title{
NAPICU position on the monitoring, regulation and recording of the extra care area, seclusion and long-term segregation use in the context of the Mental Health Act 1983: Code of Practice (2015)
}

\section{Background}

The Mental Health Act 1983: Code of Practice (MHA CoP) was updated in 2015 and the definition for seclusion was revised to the following:

'Seclusion refers to supervised confinement and isolation of a patient, away from other patients, in an area from which the patient is prevented from leaving, where it is of immediate necessity for the purposes of containment of severe behavioural disturbance which is likely to cause harm to others.' [MHA CoP (2015) para 26.103]

In certain circumstances this has resulted in use of an Extra Care Area (ECA) meeting the requirements for monitoring of seclusion. There has however been significant confusion around the monitoring of seclusion particularly for those patients who are engaged in the ECA in the company of staff. An ECA is defined as:

'a quiet, low-stimulus space for patients experiencing high levels of arousal during periods of disturbed behaviour and can be used for de-escalation, patient support and management, and treatment in a bespoke space for high intensity intervention.

The ECA should provide for the daily living needs of a single patient for a limited period. It should include:

- De-escalation and/or seclusion room;

- Toilet and shower facilities;

- Sitting room with safe furnishings'

[PICU National Minimum Standards (NMS) (2014), paras 7.2.21-7.2.22]

\section{Methods in use within mental health in-patient facilities for the management of aggression and acute}

\section{disturbance}

Practice within UK Mental Health inpatient facilities for the management of aggression and acute disturbance includes the use of:

- Traditional seclusion, i.e. a patient being locked alone in a room

- Use of an ECA, i.e. a patient separated from the rest of the unit's patient population within a specific designated area although never locked alone in a room or separated from the staff by means of a physical barrier

- A combination of both of the above approaches, i.e. where a patient may at times be locked alone in a room although at other times engaged within the ECA within which the seclusion room is often located.

\section{Position outline}


To be consistent with MHA CoP (2015) paras 26.103-26.149, the need for monitoring and regulation starts whenever a patient is:

- Locked alone in a room; monitoring starts from the point that the staff have left the room and closed the door

- Prevented from leaving a room by a fixed physical barrier and separated from the staff, e.g. a door is wedged shut by placement of foot or holding of a door handle from the other side.

\section{Use of the ECA}

- To be consistent with MHA COP (2015) paras 26.103-26.149, the need for monitoring and regulation starts whenever a patient is taken to an ECA (or any other area used as such) and would be prevented from leaving if they attempted, or expressed a wish to leave and any PMVA procedures (including verbal negotiation) having been concluded.

\section{Long-term segregation}

\section{Definition}

Long-term segregation refers to a situation where, in order to reduce a sustained risk of harm posed by the patient to others, which is a constant feature of their presentation ... a patient should not be allowed to mix freely with other patients on the ward or unit on a long-term basis. [MHA CoP (2015) para 26.150]

Long-term segregation refers to a situation in which it is foreseeable that it will be necessary to keep the patient separate from other patients, although not from the staff, for an extended period of time (more than 72 hours). The criteria applicable for considering whether the procedure for long-term segregation is appropriate include the following:

- Sustained risk to others is a constant feature of the patient's mental state

- If the patient is allowed to mix freely with other patients on the ward, other people would be at constant risk

- Short periods of ECA or traditional seclusion use combined with other treatment options would not ameliorate the risk.

\section{Characteristics}

- Long-term segregation should provide a similar arrangement to the extended use of the ECA in terms of proximity to, interaction with staff and access to areas of the ECA.

- Long-term segregation may involve more opportunities than the extended use of the ECA and may include periodic assess to other areas of the Unit or spaces away from the Unit.

- This may include eating in the dining area or visits to the general garden area or activity rooms, consistent with risk assessments.

- Patients should not feel isolated from contact with the staff (indeed it is highly likely that they should be supported through enhanced engagement) or deprived of access to therapeutic interventions while subject to long term segregation. 


\section{- napicu}

- Monitoring and regulation procedures consistent with MHA CoP (2015) paras 26.150-26.160 (Long-term segregation) should be considered whenever a patient is separated from their peers.

- In the ECA or any other area of the Unit from which the patient would be prevented from leaving for more than 72 hours, consideration should be given to assessing the patient's position in respect of the criteria for long-term segregation detailed in MHA CoP (2015) paras 26.150-26.160.

- This should include a formal review process involving the independent mental health advocate (if available), MDT, senior clinical manager or modern matron, and service commissioners etc. as laid out in MHA CoP (2015) para 26.155.

\section{Where traditional seclusion and an ECA are used in combination}

- At any time in which a patient is locked alone in a room (traditional seclusion), the requirements for monitoring, regulation and recording should always be consistent with MHA CoP (2015) paras 26.10326.149 (see 1 above).

- At times when the patient has left the seclusion room but is restricted to the ECA (and the Unit standard level of autonomy has not been returned to the patient), they should continue to be subject to monitoring, review and recording consistent with guidance on traditional seclusion (MHA CoP (2015) paras 26.103-26.149).

- If after 72 hours (or before if appropriate decisions and review processes have occurred) there is a continued need for the individual to remain separated from their peers without the Unit standard level of autonomy being returned, then an assessment should be completed with regard to the criteria for longterm segregation regulated by MHA COP (2015) paras $26.150-26.160$ (see 3 above). This should include a formal review process involving the independent mental health advocate, MDT, senior clinical manager or modern matron and service commissioners.

- If at any time during the patient's management within the definition of long-term segregation $s /$ he is returned to being locked alone in a room (traditional seclusion) then the procedures detailed within MHA CoP (2015) paras 26.103-26.149 should be restarted.

\section{Recording and monitoring}

\section{Traditional seclusion}

- Whenever a patient is locked alone in a room with staff monitoring from the other side of a locked or fixed door, then a record of the patient's behaviour should be made every 15 minutes (MHA CoP (2015) para 26.123).

\section{Extended use of the ECA and long-term segregation}

- Whenever a patient is separated from their peers in a place from which they would be prevented from leaving, although are never separated from the staff, then a summary of their behaviour should be recorded hourly (MHA CoP (2015) para 26.154). 
- Recording every 15 minutes (MHA COP (2015) para 26.123) should be avoided in circumstances where patients are not separated from the staff as this is likely to be perceived as disruptive and unhelpful by the patient.

\section{Organisational monitoring}

Mental health providers using any of the methods above should monitor the number and pattern of episodes of seclusion at organisational level for quality improvement purposes.

Within monitoring procedures, the number and duration of episodes of seclusion should be clearly identifiable between those that have involved:

- A patient being locked alone in a room (traditional seclusion)

- The use of the ECA where patients have remained in the company of staff. 